\title{
Channel-bonding CMOS transceiver for 100 Gbps wireless point-to-point links
}

\author{
José Luis Gonzalez-Jimenez ${ }^{*}$, Cédric Dehos, Nicolas Cassiau, Alexandre Siligaris, Antonio Clemente, \\ Rafaelle D'Errico, Valentin Savin, Yves Durand, Antonio De Domenico and Dominique Noguet
}

\author{
${ }^{*}$ Correspondence: joseluis. \\ gonzalezjimenez@cea.fr \\ Université Grenoble-Alpes, CEA-Leti, \\ Grenoble, France
}

\begin{abstract}
$5 \mathrm{G}$ systems and networks are expected to provide unprecedented data-rate to final users and services, in combination with increased coverage and density. The traffic generated at the edges of the network should be hauled through high capacity data-conveyors. Extremely high data-rate links able to provide optical-fiber like performance in the order of 100 Gbps are required to reduce the cost and increase the flexibility of the network infrastructure deployment. This paper presents a full transceiver architecture based on a channel-bonding radio-frequency front-end operating at millimeter-wave frequencies and digital baseband processing units able to provide such data-rates with a feasible implementation in low-cost CMOS technologies. The baseband section of the receiver includes digital compensation algorithms that allow to cope with some of the radio front-end impairments. The main functionalities of the proposed transceiver architecture are validated in hardware.
\end{abstract}

Keywords: Millimeter-wave CMOS integrated circuits, Radio transceivers, Phase noise, Digital-baseband impairments compensation, High-data rate wireless links

\section{Introduction}

Several millimeter wave (mmW) frequency bands located from $24 \mathrm{GHz}$ up to $40 \mathrm{GHz}$, depending on the country, are currently under consideration according to 3GPP Release 15 on 5G-NR standard, to provide enhanced mobile broadband (eMBB) access services, but also for backhaul and fronthaul point-to-point links and for internet to the home deployment. However, the available bandwidth at those frequencies will not allow the optical-fiber compatible data rates required to support the increase in data traffic that is expected from $5 \mathrm{G}$ and beyond-5G networks. Low installation costs can be achieved by using short and medium-range wireless links able to provide 100 Gbps rank data rates that can compete with optical fiber. Currently, several agencies such as ETSI, FCC, and MICI [1] are considering the bands beyond $95 \mathrm{GHz}$ in order to provide such class of high-data rate for short and medium distance fixed links.

There exist only a few ways to increase the data rate of a wireless link when considering just the electronic transceiver (i.e., in spite of using spatial and/or polarization diversity): increasing the signal bandwidth, increasing the modulation order, improving

(c) The Author(s). 2020 Open Access This article is licensed under a Creative Commons Attribution 4.0 International License, which permits use, sharing, adaptation, distribution and reproduction in any medium or format, as long as you give appropriate credit to the original author(s) and the source, provide a link to the Creative Commons licence, and indicate if changes were made. The images or other third party material in this article are included in the article's Creative Commons licence, unless indicated otherwise in a credit line to the material. If material is not included in the article's Creative Commons licence and your intended use is not permitted by statutory regulation or exceeds the permitted use, you will need to obtain permission directly from the copyright holder. To view a copy of this licence, visit http://creativecommons.org/licenses/by/4.0/. 
to a certain extent the signal to noise ratio (SNR), or a combination of these factors. The SNR at the receiver is governed by the transmit power, the channel path loss, the antenna gains (at transmitter and receiver), and the noise figure of the receiver. Going up in carrier frequency allows to access larger portions of the available spectrum. However, for a given carrier frequency, it is difficult to implement power-efficient radio frequency (RF) electronics having more than $20 \%$ of relative system bandwidth (BW). Taking this constraint into account, Fig. 1 presents the maximum capacity as dictated by Shannon-Hartley information theorem on channel capacity for a set of reasonable link parameters in terms of transmit power at the input of the antenna, receiver noise figure and transmit $(\mathrm{Tx})$, and receive $(\mathrm{Rx})$ antenna gains. The figure shows the peak data rates that can be achieved for three distances $(10 \mathrm{~m}, 50 \mathrm{~m}$, and $100 \mathrm{~m})$ considering a line-of-sight path loss model, an additive white Gaussian noise (AWGN) channel and data rates achievable with several modulation types for $20 \%$ relative BW. Below the peak, the channel capacity is limited by available BW, and beyond the peak it is limited by the noise. The raw data rate that is provided by each modulation type is shown as well. For $100 \mathrm{~m}$ links, $100 \mathrm{Gbps}$ data rate is the maximum achievable for the set of parameters displayed in the inset; it requires a carrier frequency of $125 \mathrm{GHz}$ with $25 \mathrm{GHz}$ of available BW. The position of the peak data rate at a given distance depends on the link parameters. For example, if the Tx power is increased by $10 \mathrm{~dB}, 240 \mathrm{Gbps}$ at $100 \mathrm{~m}$ are theoretically possible using $60 \mathrm{GHz} \mathrm{BW}$ around $300 \mathrm{GHz}$. Interestingly enough, the peak data-rate is achieved always for 4 bits $/ \mathrm{Hz}$ modulation schemes, such as 16

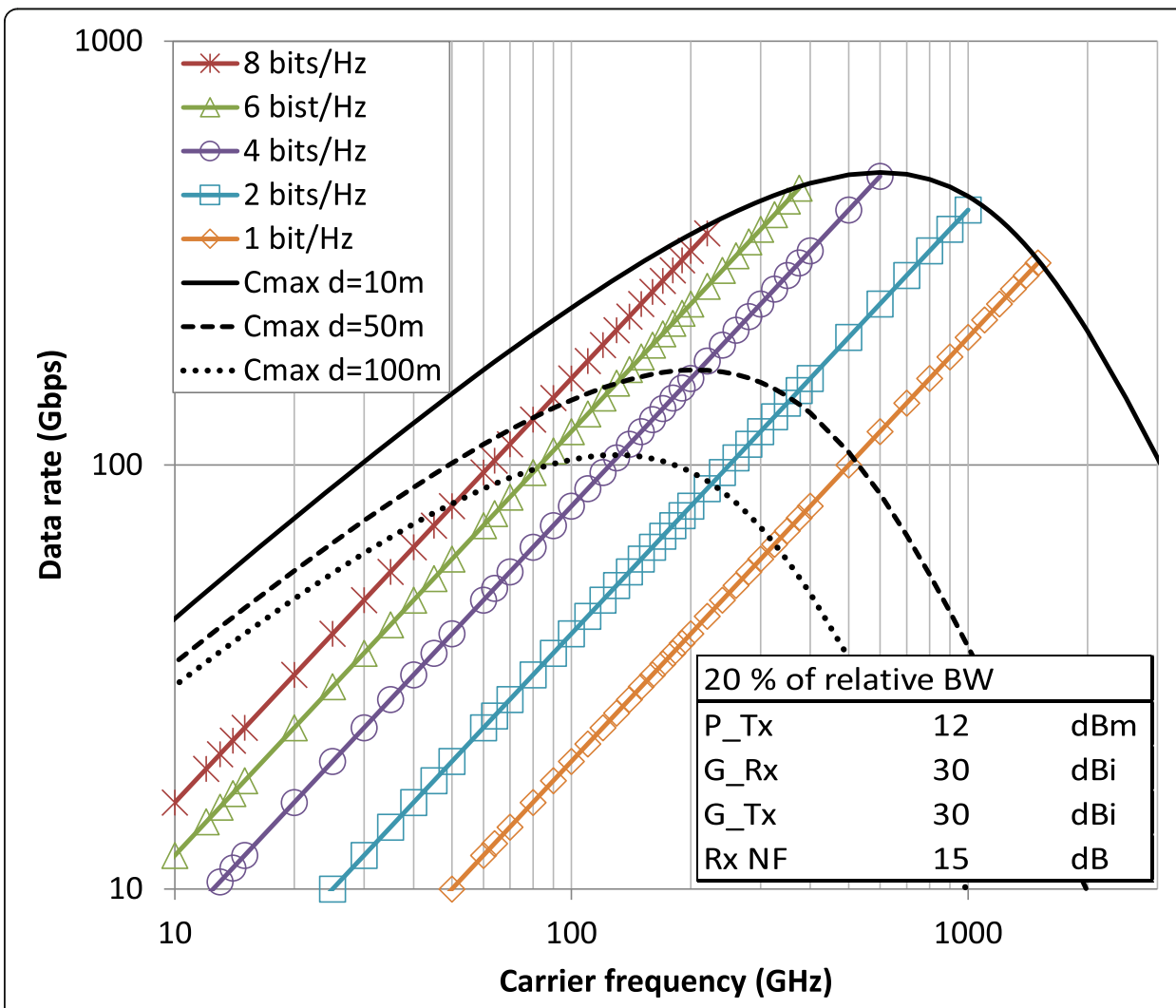

Fig. 1 Maximum theoretical data rate for a constraint of available bandwidth equal to 20\% of the carrier frequency for different link distances and fixed set of transceiver parameters in AWGN channels 
quadrature amplitude modulation (QAM). Another important conclusion is that in order to achieve 100 Gbps and beyond data rates, carrier frequencies in the range 120$300 \mathrm{GHz}$ need to be used with RF link bandwidths in the order of a few tens of $\mathrm{GHz}$.

The challenges of providing several tens of Gbps wireless links have been addressed by the scientific community following two different implementation approaches. The main characteristics of recent III-V [2-10], complementary metal-oxide semiconductor (CMOS)/bipolar CMOS (BiCMOS) [11-21], and photonic based [22, 23] mmW multiGbps wireless transceivers are shown in Fig. 2. The advantage of III-V technologies is based on their higher $f_{\max }$ compared to CMOS or BiCMOS technologies. With this technology a large amount of RF bandwidth in a single RF channel is typically used
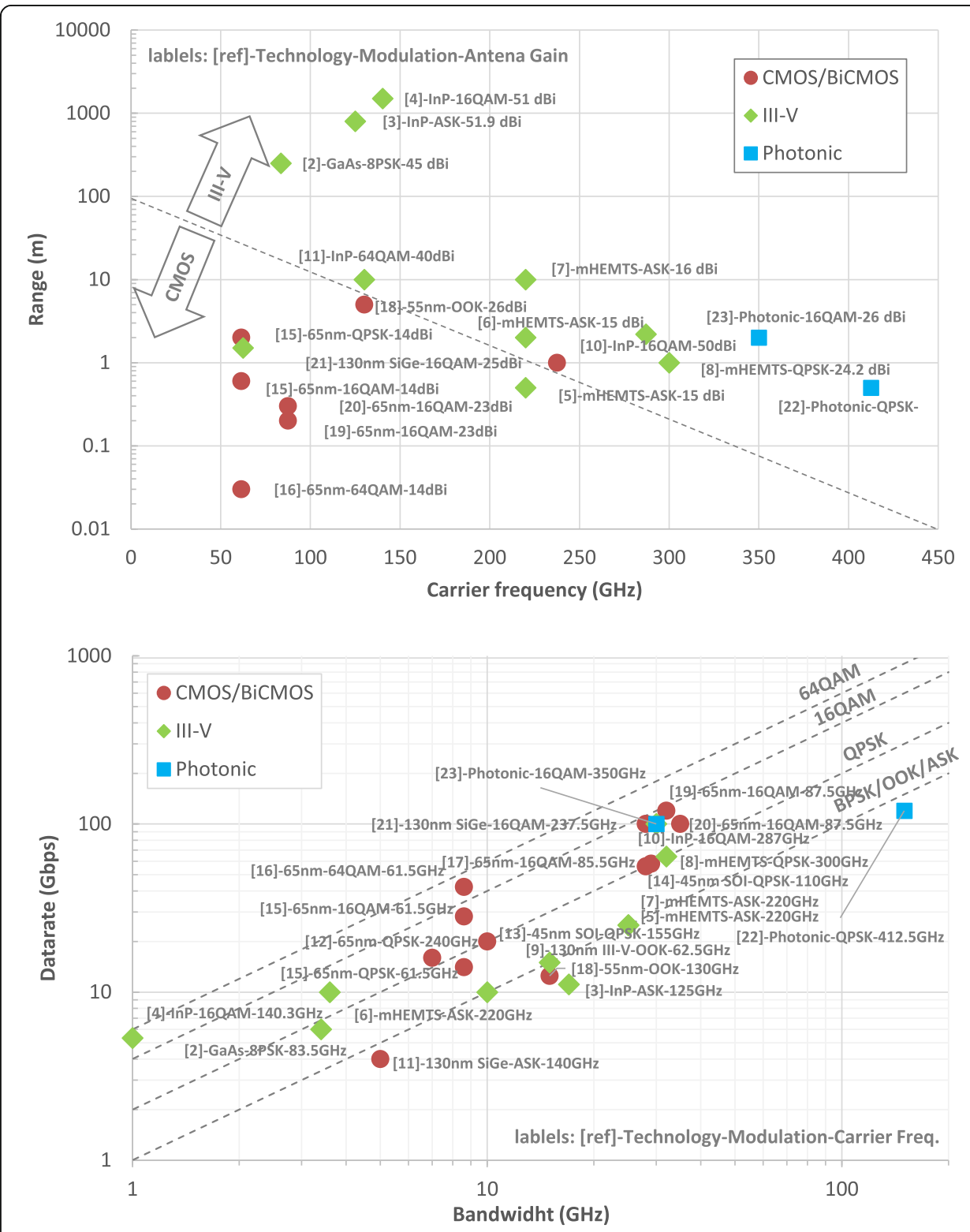

Fig. 2 Recent CMOS/BiCMOS, III-V, and photonic multi-Gbps transceiver: range vs carrier frequency (left) and data-rate vs bandwidth (right). The labels include information about technology, modulation type, and antenna gain and carrier frequency, when available 
with simple modulation schemes such as amplitude-shift keying (ASK)/binary phaseshift keying (BPSK) which allow for digital-less demodulation. Such transceivers have been demonstrated for carrier frequencies up to $300 \mathrm{GHz}$ and achieve communication in the range of $1 \mathrm{~m}$ up to $1 \mathrm{~km}$ using high gain antennas, as shown in Fig. 2. The second approach is based on CMOS or BiCMOS technologies that are limited to lower frequencies and bandwidths. They compensate the limited BW by exploiting higherorder modulations such as quadrature PSK (QPSK), 16QAM, or even 64QAM. This second type of transceivers have demonstrated high data rates at lower carrier frequencies, comparable to those of much larger bandwidth III-V or photonic based transceivers, as shown in Fig. 2. Provided that perfect synchronization and channel equalization is achieved, the optimal modulation appears to be 16QAM for a system exploiting a BW of $20 \%$ of the central frequency on AWGN channels, in agreement with the theoretical result shown in Fig. 1. It shall be mentioned that although the AWGN condition over $20 \%$ bandwidth is very unrealistic below $10 \mathrm{GHz}$, recent channel measurements have shown that the channel is hardly affected by multipath at very high frequencies [24].

One characteristic that the most efficient state-of-the art CMOS transceivers have in common is the use of multiple parallel RF channels (i.e., channel-bonding) in order to relax the signal bandwidth vs the operating frequency $[16,17,19,20]$, a characteristic that is shared with some of the photonic-based transceivers [22]. Indeed, even though high sampling frequency data converters exist to interface multi-GHz BW front-ends with their digital baseband processing units, their power consumption increases exponentially with sampling frequency beyond 0.5-1 Gsample/s, as shown in [25]. Digital baseband systems data-rate processing capabilities are somehow limited to a few Gbps, and are also bonded by power consumption. Hence, a reasonable trade-off needs to be done between each individual channel bandwidth and the overall number of channels required to cover the full RF band and provide the desired total throughput.

The paper describes an investigation on a multi-channel transceiver architecture able to provide $100 \mathrm{Gbps}$ data rate by bonding several base-band (BB) channels of reasonable bandwidth. Section 2 presents the overall architecture detailing a suitable frequency plan, the radio front-end, and the base-band processor. Section 3 describes the main impairments of large-bandwidth channel-bonding radio front ends, such as phase noise, intra-channel interferences, I/Q imbalance, non-linearities, and the mitigation mechanisms that can be implemented in the BB to compensate such RF imperfections. The methodology used to investigate the effectiveness of the proposed compensation mechanisms based on link-level simulations and measurements on a hardware implementation of the transceiver on a laboratory validation platform is presented in section 4. This study allows to derive reasonable values for the RF front-end (RFFE) imperfections that can be compensated digitally by the BB section. The main lessons learned from this study are summarized in section 5 that concludes the paper.

\section{Transceiver architecture}

With the goal of implementing fully integrated 100 Gbps transceivers, multi-channel (or channel-bonding) architectures are considered in this paper as the best option to achieve a large overall RF bandwidth with moderately complex modulations (i.e., 16QAM) along with reasonable baseband analog and digital bandwidth. 


\subsection{Frequency plan}

Figure 3 illustrates a channel bonding strategy offering $N$ channels at RF. Two-step frequency conversion is considered in order to avoid offset problems conveniently distribute the gain across the various transceiver stages. Channel-bonding is applied both at an intermediate frequency (IF) and at RF, in order to optimize the frequency plan and the number of local oscillator signals required.

One of the biggest challenges of channel-bonding architectures is the generation of the several required local oscillator (LO) signals. In the example shown in Fig. 3, for a $N$ channel-bonding transceiver, if there are $K$ channels in each IF lane, a total of $N / K$ lanes are required, so that $K$ LO frequencies are needed for up and down conversion from/to $\mathrm{BB}$ to/from IF and $N / K$ LO frequencies are needed for the IF to RF (or vice versa) conversion. In the proposed RFFE architecture, detailed in the next section and shown in Fig. 5, it has been chosen $N=16$ and $K=4$, which results on 8 different LO frequencies.

A frequency plan, shown in Fig. 4, is proposed in this work to allow all the LO frequencies to be generated from a single common clock reference using high order frequency multiplication. This technique is based on pulsed oscillators followed by injection-locked oscillators (ILOs) that filter out the desired single tone LO signal as explained in [26]. In the proposed architecture, the IF LO frequencies are $\{46 ; 48 ; 50 ; 52\}$ GHz. They are generated using multiplication by $\{23 ; 24 ; 25$; 26\} from a $2 \mathrm{GHz}$ input signal. The RF LO frequencies are $\{80 ; 88 ; 96 ; 104\} \mathrm{GHz}$, and are generated using frequency multiplication by $\{20 ; 22 ; 24 ; 26\}$ from the same $2 \mathrm{GHz}$ input signal followed by, for example, a push-push based frequency doubler added after the filtering ILOs. The $2 \mathrm{GHz}$ frequency value corresponds as well to the channel spacing, since all LO frequencies are integer multiples of this value. Note that the overall link consists of $16 \times 2 \mathrm{GHz}$ channels, resulting in $32 \mathrm{GHz}$ of RF bandwidth centered around $141 \mathrm{GHz}$.

\subsection{Link budget and RFFE architecture}

Figure 5 shows a proposed super-heterodyne channel-bonding RFFE architecture based on the abovementioned frequency plan and the resulting 16 channels spaced by $2 \mathrm{GHz}$.

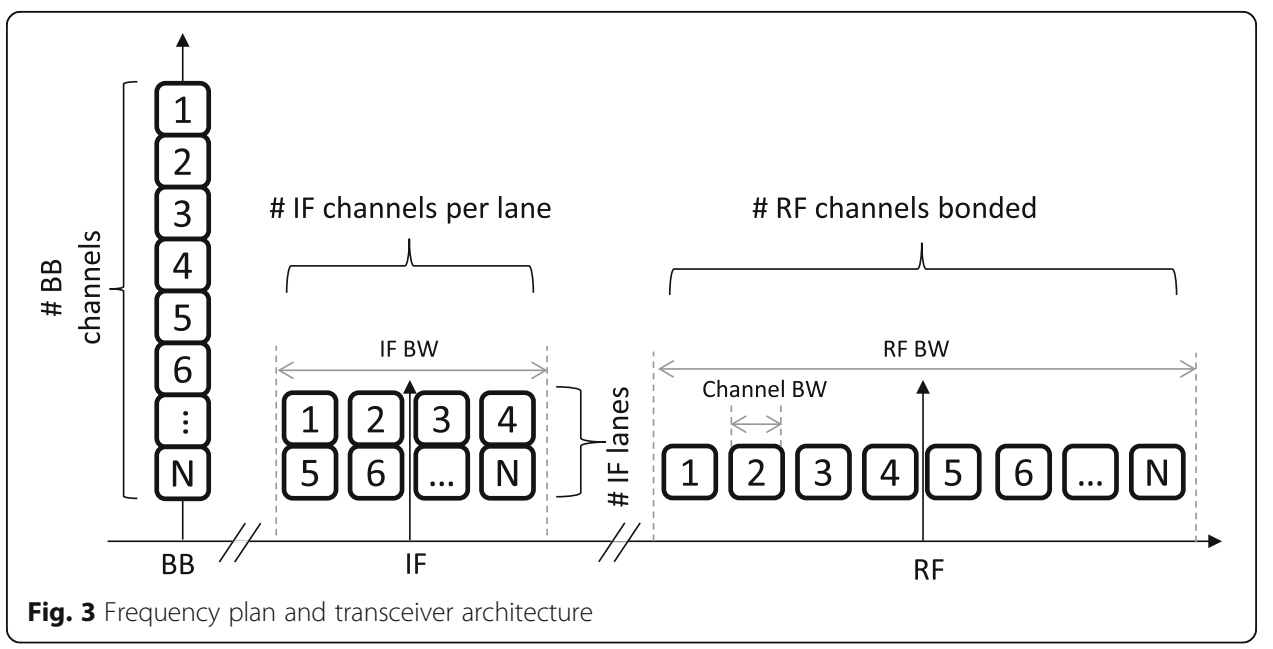




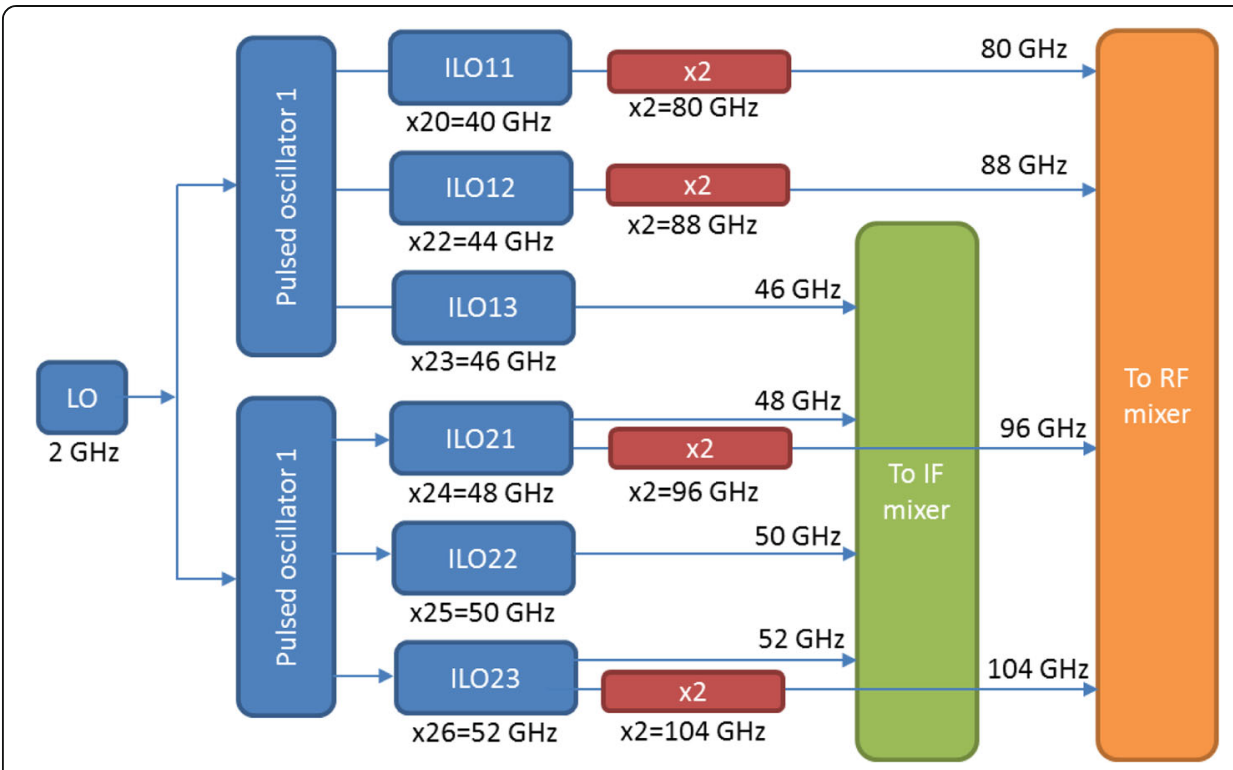

Fig. 4 LO signal generation schema

The useful bandwidth per channel is considered to be of $1.6 \mathrm{GHz}(400 \mathrm{MHz}$ of guardband is left between adjacent channels to alleviate channel filters and non-linearity constraints). In the transmitter, the $16 \mathrm{BB}$ channels are grouped into sets of four. Each of the $\mathrm{BB}$ channel of a set is up converted to a different IF. Then the channels are bonded together in the band 45-53 GHz. The four IF lanes share the same four LO frequencies for BB to IF up conversion. Then, the four IF bundles are up converted to D band using a different LO frequency for each one. After this second frequency conversion, the four up-converted IF lanes are combined in RF frequency resulting in a total of 16 bonded channels at D band $(125-157 \mathrm{GHz})$. The receiver is organized in a complementary way, as shown in the figure. This two-step mixing strategy allows to relax the gain and bandwidth constrains for the RF circuits. At the transmitter side, power amplifiers operating at IF have $14 \%$ relative $\mathrm{BW}$ and those on sub-bands at RF have $6 \%$ relative BW. At the receiver side, a single full-band low noise amplifier (LNA) is placed at the antenna port with limited gain to optimize the noise figure, following by RF and IF amplifiers with reduced BW constraints. Because the LNA receives the full bandwidth, the $20 \%$ carrier frequency assumption of Fig. 1 is preserved.

A link budget calculation was done (see Table 1) for the link considered in this work, using the following assumptions on $\mathrm{Tx}$ and Rx building blocks based on existing CMOS circuit data:

The four power amplifiers (PA) operating in $\mathrm{D}$ band shall provide $10.5 \mathrm{dBm}$ output 1 $\mathrm{dB}$ compression point $(\mathrm{OCP} 1 \mathrm{~dB})$ over their $6 \%$ relative bandwidth. This level of output power is reported in [27].

The antenna scheme shall provide $33 \mathrm{~dB}$ gain in the $32 \mathrm{GHz}$ bandwidth. For this purpose, a transmit array antenna of $40 \times 40$ elements (panel of $25 \mathrm{~cm}^{2}$ ) can be used [28], assuming $40 \%$ transmission efficiency.

At the receiver side, the LNA shall provide at least $12 \mathrm{~dB}$ gain over $32 \mathrm{GHz}$ bandwidth and a noise figure of $9 \mathrm{~dB}$. The total noise figure of the receiver can be then limited to $12 \mathrm{~dB}$. One LNA fulfilling this requirement can be found in [29]. 


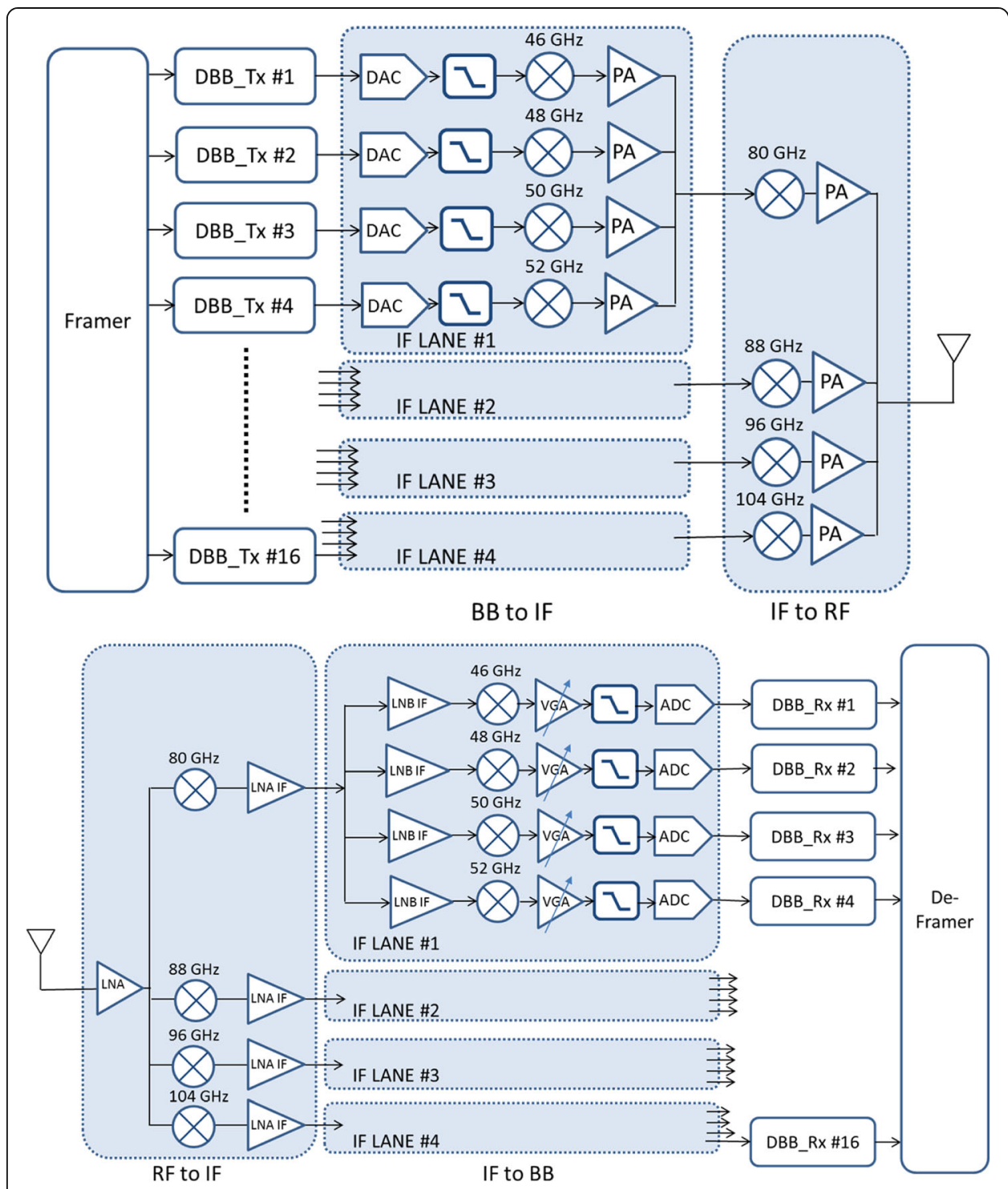

Fig. 5 Proposed multi-channel (or channel-bonding) 100 Gbps transceiver architecture

The IF stages at both the transmitter and the receiver shall tackle the same constraints in terms of power, gain, bandwidth, linearity as the $60 \mathrm{GHz}$ transceivers developed for WiGig applications [30].

On-chip combiners/splitters are required able to combine up to four D band channels with reasonable insertion loses. One possible implementation of this type of combiner/splitter has been recently reported in [31].

According to the results shown in Table 1, a raw data rate of 102.4 Gbps can be achieved at a maximum of $50 \mathrm{~m}$ by using 16QAM modulation.

\subsection{Digital baseband architecture}

Single carrier (SC) waveform with frequency domain equalization (FDE) [30] is one of the possible candidate signals for the multi-channel transceiver. It has been selected 
Table 1 Link budget calculation (most numbers are per BB channel)

\begin{tabular}{|c|c|c|c|c|}
\hline Element & 64QAM & 16QAM & QPSK & BPSK \\
\hline \multicolumn{5}{|l|}{ Emitter } \\
\hline RF BW $(\mathrm{GHz})$ & 32 & 32 & 32 & 32 \\
\hline Number of bonded channels & 16 & 16 & 16 & 16 \\
\hline Single channel BW (GHz) & 1.6 & 1.6 & 1.6 & 1.6 \\
\hline Per PA OCP1B (dBm) & 10.5 & 10.5 & 10.5 & 10.5 \\
\hline Per ch. OCP1 dB (dBm) & 4.5 & 4.5 & 4.5 & 4.5 \\
\hline Back off (dB) & 7 & 5 & 3 & 3 \\
\hline Average power @PA out (dBm) & 3.5 & 5.5 & 7.5 & 7.5 \\
\hline Per channel Tx av. Pout (dBm) & -2.5 & -0.5 & 1.5 & 1.5 \\
\hline Interconnection losses (dB) & 2 & 2 & 2 & 2 \\
\hline Antenna gain (dBi) & 33 & 33 & 33 & 33 \\
\hline $\operatorname{EIRP}(\mathrm{dBm})$ & 28.5 & 30.5 & 32.5 & 32.5 \\
\hline \multicolumn{5}{|l|}{ Channel } \\
\hline Range (m) & 25 & 50 & 120 & 150 \\
\hline Attenuation coefficient & 2 & 2 & 2 & 2 \\
\hline Carrier frequency (GHz) & 156 & 156 & 156 & 156 \\
\hline $\mathrm{O}_{2} \&$ rain attenuation $(\mathrm{dB})$ & 0.80 & 1.60 & 3.84 & 4.80 \\
\hline Path loss (dB) & -105.06 & -111.88 & -121.73 & 124.63 \\
\hline \multicolumn{5}{|l|}{ Receiver } \\
\hline Antenna gain (dBi) & 33 & 33 & 33 & 33 \\
\hline Interconnection losses (dB) & 2 & 2 & 2 & 2 \\
\hline Power per channel (dBm) & -44.6 & -49.5 & -57.3 & -60.2 \\
\hline Power at LNA input (dBm) & -32.6 & -37.5 & -42.3 & -48.2 \\
\hline$N F(d B)$ & 12 & 12 & 12 & 12 \\
\hline Implementation loss margin (dB) & 4.2 & 2.5 & 1.2 & 1.3 \\
\hline SNR @ ADC in (dB) & 25.3 & 20.5 & 12.7 & 9.8 \\
\hline SNR for PER $10^{-5}(\mathrm{~dB})$ & 21 & 18 & 11.5 & 8.5 \\
\hline Raw data-rate (Gbps) & 153.6 & 102.4 & 51.2 & 25.6 \\
\hline
\end{tabular}

due to its low peak to average power ratio at the transmitter and by the ease of correction of phase rotations at the receiver. Furthermore, FDE allows for inter-symbol interference mitigation with a complexity similar to orthogonal frequency division multiplexing (OFDM).

The structure of the frame is illustrated in Fig. 6. This structure is mainly motivated by the need for time synchronization and RF impairments estimation. Time synchronization uses a known preamble made of $N_{S}$ repetitions of a given sequence. An autocorrelation and peak detection process allows to find the synchronization sample with very low probabilities of non-detection and false alarm. This preamble also aims at measuring the carrier frequency offset (CFO) (see below). The second preamble is made of $M$ repetitions of a known sequence. It is dedicated to the IQ mismatch estimation in the time domain (see below). The third preamble allows for channel estimation. The payload is made of a number of symbols (the length of a symbol corresponds to the fast fourier transform (FFT) size of the frequency domain equalizer) where pilots (known samples) are distributed. 


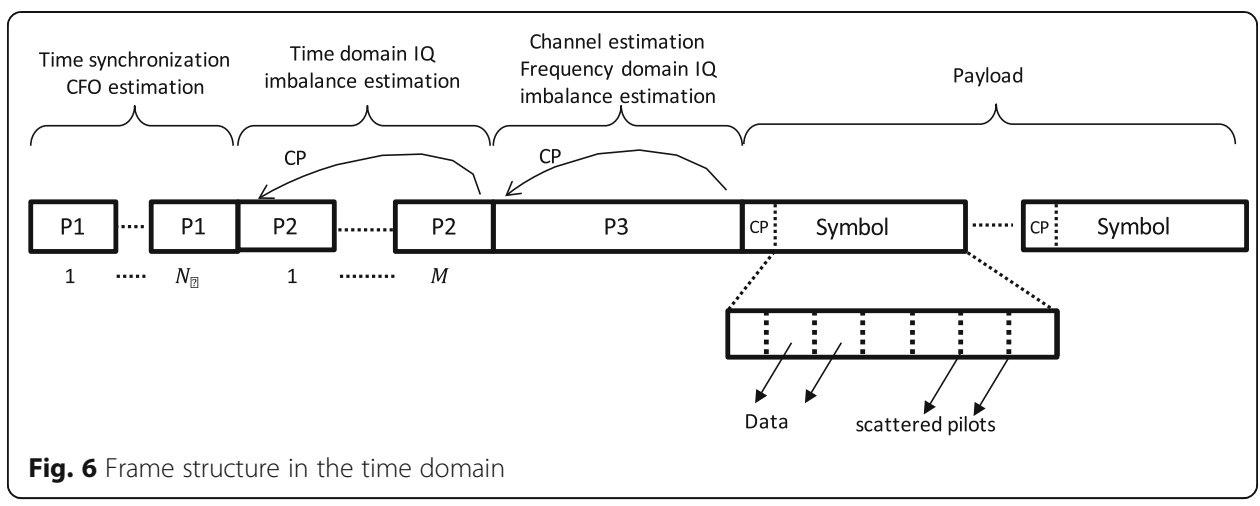

\section{RFFE impairments modeling and compensation}

The feasibility of the proposed channel-bonding architecture relies on implementing BB digital compensation techniques able to compensate the "imperfections" from the RFFE, alleviating in this way the performance required for the various high-frequency building blocks. A comprehensive model of the full transceiver composed of the BB processor and the RFFE for the Tx and the Rx, along with a realistic channel model derived from experimental measurements [32], is used to validate the transceiver. In this section the main impairments of the RFFE are reviewed. For each one, the way it is included in the link-level model of the transceiver and the numerical values of the different parameters involved are presented and then the compensation mechanism is briefly described.

\subsection{Channels interferences and LO leakage}

Channel to channel interferences are a serious concern in channel-bonding transceivers. In the case of the proposed architecture, the situation is exacerbated because each BB channel shares the same IF frequency as three other BB channels. This is modeled through three coupling coefficients $\left(\beta_{1}, \beta_{2}, \beta_{3}\right)$, one for each of the interfering IF channel. Furthermore, the technique used to generate the LO from a lower frequency signal that matches the channel spacing is also prone to generating LO spurs at the adjacent channel LO frequencies. Figure 7 illustrates these two channel-to-channel interference mechanisms that happen in the IF section of the transceiver. In the RF section, LO spurs in adjacent channel frequencies could be a source of channel-to-channel interference as well. All such interferences are modeled with coupling coefficients. The coefficients for adjacent channel leakage $\left(\alpha_{\mathrm{IF}}, \alpha_{\mathrm{RF}}\right)$ are calculated considering $\mathrm{LO}$ spurs of $-20 \mathrm{dBc}$ at the adjacent channel frequencies both for the IF and RF LO signals. For the co-channel interferences, the coupling coefficient $\beta_{1}$ for the closest IF lane (in terms of layout floorplan) is larger than those of the other ones $\left(\beta_{2}, \beta_{3}\right)$. In this paper it is considered that they range from -20 to $-26 \mathrm{~dB}$.

\subsection{Phase noise}

\subsubsection{Modeling}

Phase noise (PN) is modeled according to the model proposed in [33]. Parameters that describe the phase noise spectrum are illustrated in Fig. 8: (i) $1 / \mathrm{f}^{2}$ phase noise at $F_{\text {ref }}$ (the spectrum shape at this point decreases by $20 \mathrm{~dB}$ per decade); (ii) PLL cut-off frequency; (iii) phase noise floor, i.e., the white phase noise (WPN) floor of the oscillator. Phase noise and white phase noise occur at each stage of the transmitter and receiver 


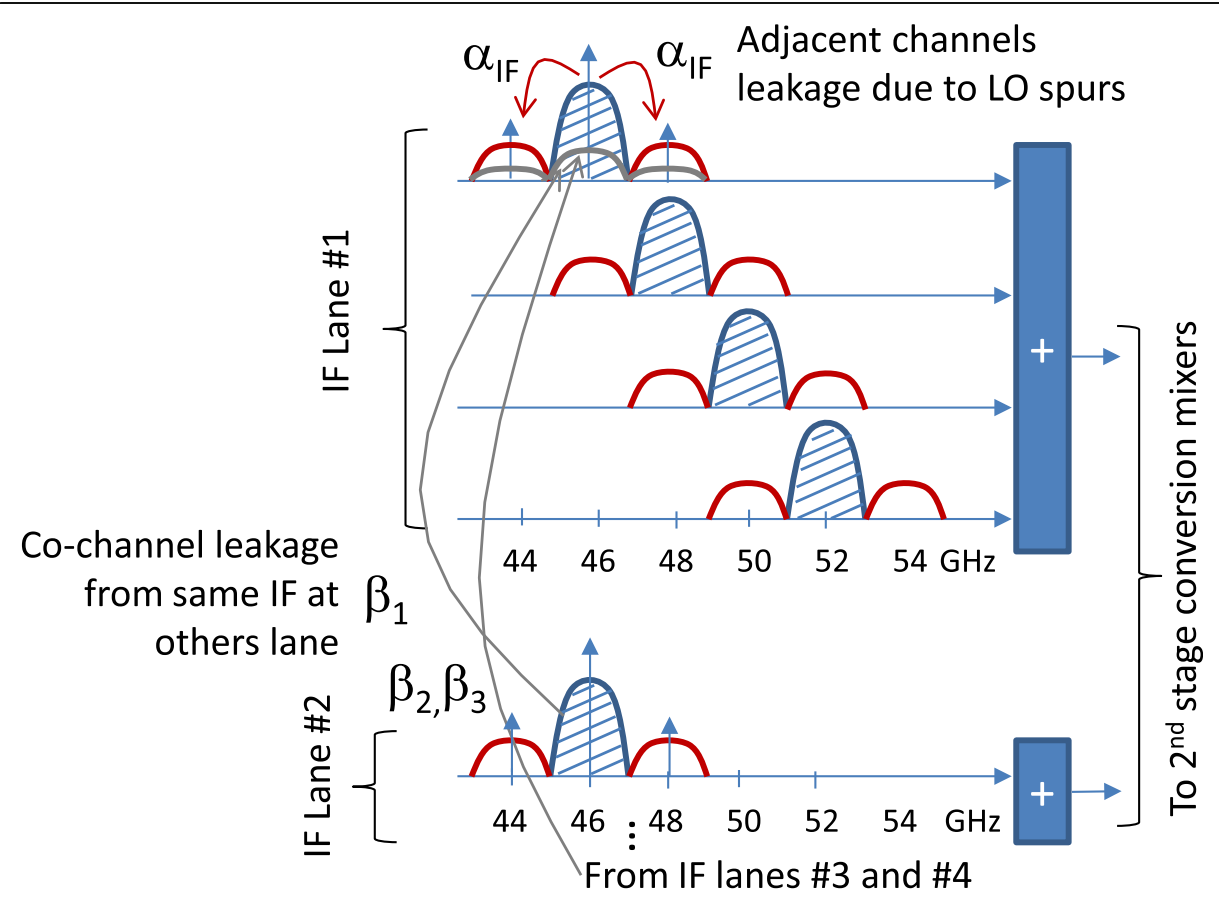

Fig. 7 Channel to channel interference mechanisms

where mixers are involved, i.e., at the output of each ILO and $\times 2$ multiplier in Fig. 4 . In the link-level simulations, the $1 / \mathrm{f}^{2}$ (bilateral) phase noise of the $2 \mathrm{GHz}$ LO is -130 $\mathrm{dBc} / \mathrm{Hz}$ (at $\mathrm{F}_{\text {ref }}=1 \mathrm{MHz}$ ). The WPN of the $2 \mathrm{GHz}$ LO is found to be limited to -154 $\mathrm{dBc} / \mathrm{Hz}$ for $16-\mathrm{QAM}$. The WPN at the output of ILOs and at the output of $\times 2$ multipliers is set to $-124 \mathrm{dBc} / \mathrm{Hz}$. The PLL cut-off frequency is selected to be $250 \mathrm{kHz}$.

\subsubsection{Compensation}

Phase rotations due to phase noise and residual CFO are estimated and corrected in the receiver thanks to distributed pilots in the time domain, see Fig. 6. The phase of the known pilots is estimated at pilots' positions and interpolated at all the constellation symbols positions. Constellations symbols are then de-rotated according to the opposite of the estimated phase.

\subsection{I/Q imbalance}

\subsubsection{Modeling}

IQ mismatch (also called IQ imbalance) arises when a mixer does not respect the amplitude balance or the orthogonality between the $I$ and $Q$ branches. The signal $r_{I Q}$ impacted by IQ mismatch can be written as $r_{I Q}=\alpha r+\beta r^{*}$, with $r$ as the signal without IQ mismatch, $\alpha=\cos \left(\Delta_{\phi}\right)+j \epsilon \sin \left(\Delta_{\phi}\right), \beta=\epsilon \cos \left(\Delta_{\phi}\right)-j \sin \left(\Delta_{\phi}\right), \Delta_{\phi}$ is the phase imbalance and $\epsilon$ is the gain imbalance. In the simulations $\Delta_{\phi}=5^{\circ}$ and $\epsilon=1 d B$.

\subsubsection{Compensation}

The estimation of IQ mismatch is closely related to CFO. Indeed, CFO degrades the estimation of IQ mismatch and vice-versa. Therefore, in the receiver, the algorithm in 


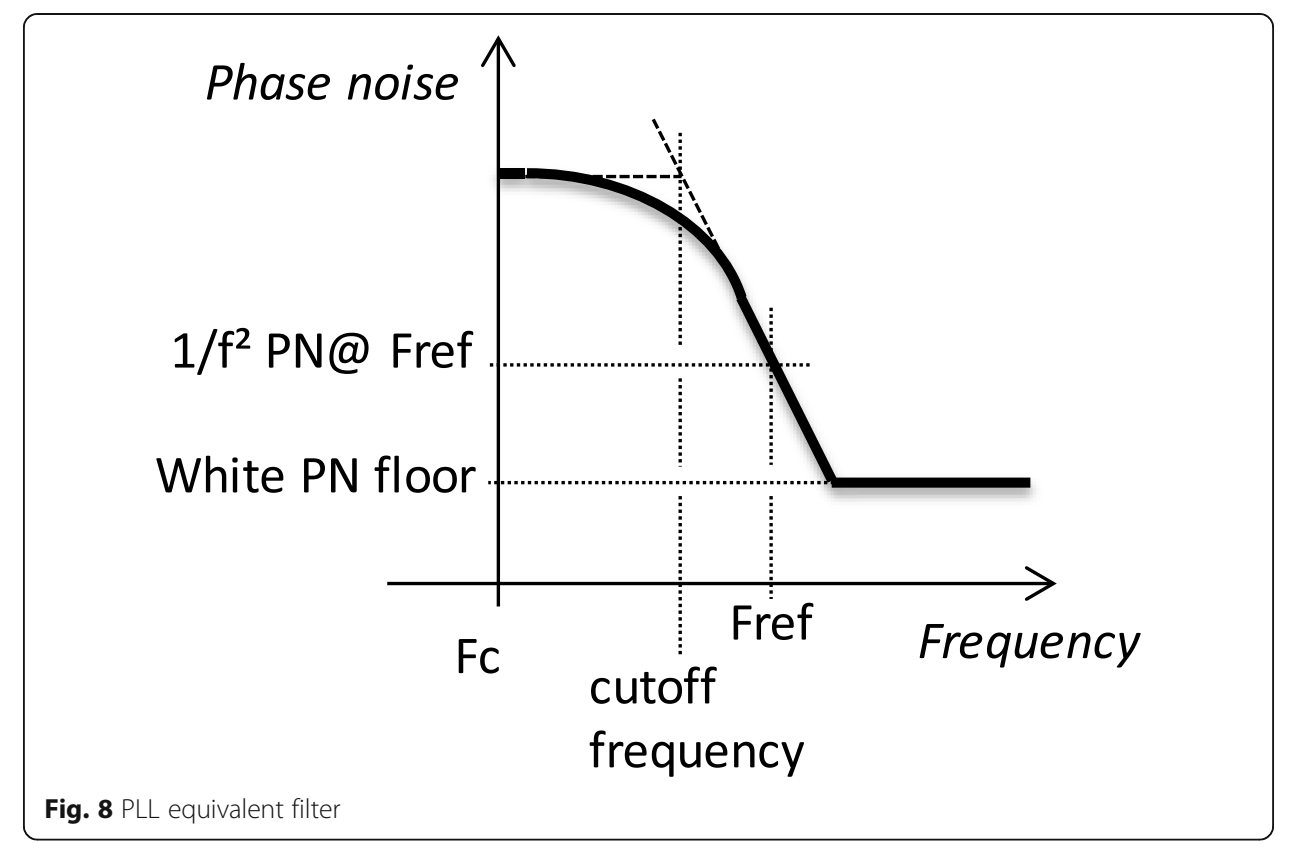

[34] has been implemented, as shown in Fig. 9. This algorithm estimates the IQ mismatch either in time domain or in the frequency domain, depending on the estimated CFO value. Furthermore, as IQ mismatch is a slowly varying impairment, it is possible and useful to use the IQ mismatch estimation computed at frame $n$ to correct the time synchronization preamble at frame $n+1$.

\subsection{Non-linearities}

The RF components that may introduce non-linearities are mixers, power amplifiers, LNA, and LNB. Non-linearity of a component may cause signal saturation and therefore a packet error rate (PER) increase. For link-level simulations, the gain and the

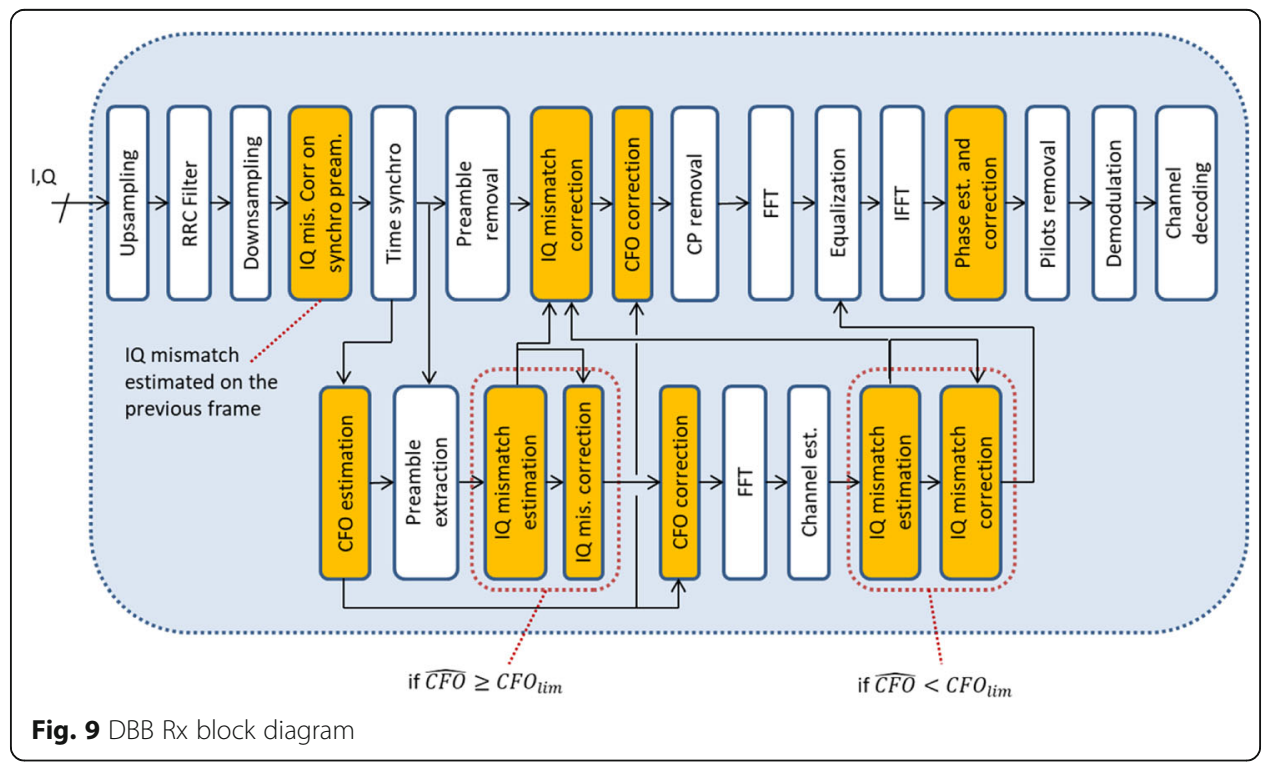


third-order input interception point (IIP3) (or the $1 \mathrm{~dB}$ compression point, $\mathrm{P} 1 \mathrm{~dB}$, for the PA) of components are taken into account to model their imperfect response. The parameters, extracted from previous experience [35], are summarized in Table 3 along with other system parameters.

\subsection{Carrier offset}

\subsubsection{Modeling}

$\mathrm{CFO}$ is due to the mismatch between carrier frequencies of the transmitter and the receiver. The $\mathrm{CFO}$ is expressed in $\mathrm{Hz}\left(C F O_{H z}\right)$ or in parts per million $\left(C F O_{p p m}\right)$, with $C F O_{\text {ppm }}=C F O_{H z} / F_{c} 10^{6}$. In the link-level simulations, $\mathrm{CFO}$ is modeled by a frequency shift of $2 \mathrm{CFO}_{\mathrm{Hz}}$ (times 2 in order to model the maximal error between transmitter and receiver) in each mixer of the receiver with $F_{c}=\{80,88,96,104\} G H z$ for the conversion to $\mathrm{RF}$ and $F_{c}=\{46,48,50,52\} \mathrm{GHz}$ at IF.

\subsubsection{Compensation}

The CFO estimation algorithm relies on the time domain synchronization algorithm because the CFO results in a ramp of phase in time, an estimation is given by the phase of the autocorrelation at the detection point. With such an algorithm, the maximum CFO that can be estimated, $C F O_{p p m}=20$, hereafter, is limited by the length of the autocorrelation window (128 samples). Residual CFO is corrected by time-domain distributed pilots.

\subsection{Channel estimation}

FDE requires an estimation of the channel in the frequency domain. The algorithm is based on a known transmitted preamble [36] shown in Fig. 6 (the channel is considered invariant during the frame). The channel estimate is obtained by the element-wise division of the received preamble by the known transmitted preamble. Low pass filtering of the frequency domain estimation improves the performance by reducing the contribution of channel noise and white phase noise.

It must be noted that CFO and IQ mismatch estimations have a role to play in the channel estimation process:

- If the estimated CFO is higher than a pre-determined threshold, the IQ mismatch is estimated before channel estimation, thanks to the dedicated preamble. CFO and IQ mismatch are compensated on the channel estimation preamble before channel estimation.

- In the other case, only the CFO is compensated on the channel estimation preamble before channel estimation. The IQ mismatch is estimated in the frequency domain, using the channel estimate: the IQ mismatch parameters that minimize the mean square error between consecutive IQ mismatch corrected channel coefficients are selected. Indeed, the algorithm is based on the information that the corrected channel response should be smooth.

\section{Results and discussion}

\subsection{Methodology}

The study presented in this paper relies on a combination of software and experimental equipment-based simulations. The different elements of the transceiver architecture 
presented in the previous sections have been implemented in a software simulation chain (run with Matlab), including baseband equivalent models for the radio sections. The models for the analog sections of the radio transceivers include non-idealities such as mismatches between the I and Q branches of mixers, non-linearity in the amplifiers, sideband spurs, and phase noise on the oscillators, etc., as previously described. A realistic model for the wireless channel is also implemented based on previously measured propagation characteristics at $\mathrm{D}$ band. The simulation methodology is as follows: the full transceiver software is run on a PC and the non-idealities parameters are varied in order to investigate their impact on the link-level performance. The highest frequency section of the radio is next implemented on a laboratory bench using commercial equipment and the same tests of link-level performance are repeated with a combination of software running the digital signal processing sections of the transceiver and actual equipment implementing the radio section and the propagation channel.

\subsection{Analyses of the different RF impairments on the link budget}

The model of the link implemented on the simulator includes the digital $\mathrm{BB} \mathrm{Tx}, \mathrm{a}$ model of the RFFE Tx, a realistic model of the channel at D-band derived from the measurements reported in [24], a realistic model of the Rx RFFE including imperfections as well as the Rx digital $\mathrm{BB}$. The software description of the transceiver model constitutes a link-level simulation platform. It has been used to validate the impact of the various system impairments and the ability of the compensation algorithms to correct them and achieve the target error rate. The link-level simulation platform is also used to switch ON or OFF some of the modeled impairments and assess their impact in terms of bit error rate (BER) or PER for the target SNR range considered in the link budget calculation of Table 1. Some results are shown in Fig. 10 for 16QAM modulation and typical link parameters are shown in Table 1. Two imperfections are analyzed in this example: the impact of going from 1 to 16 channels, which allows to investigate the impact corresponding to channel-to-channel interference explained in sub-section 3.1, and CFO that is varied from 1.5 to $15 \mathrm{ppm}$ and used to validate the phase tracking capabilities of the Rx digital BB (DBB) synchronization algorithms. Note that a CFO of $1.5 \mathrm{ppm}$ is the worst case, because this value is close to the $\mathrm{CFO}_{\lim }$ defined in Fig. 9. BER curves are traced for these cases and compared to the reference scenario in which no impairment is considered and the channel estimation of the $\mathrm{Rx}$ is perfect. The overall implementation loss when all impairments (and the corresponding compensation algorithms) are $\mathrm{ON}$ results in $6 \mathrm{~dB}$ for a BER before decoding of $10^{-2}$. Channel interferences, for example, account for less than $0.5 \mathrm{~dB}$ of implementation loss.

In order to gain insight on the rest of impairments, a more exhaustive analysis is done by comparing how much of the total implementation loss is due to a given impairment. This is done by turning OFF that particular impairment and leaving ON all the others. The results of this study are summarized in Tables 2 and 3 summarizes the numeric values of the various non-idealities considered in the transceiver architecture. Please note that one must carefully take conclusions from considering some of the impairments individually, since some have a correlated effect. For example, the impact of phase noise is affected by the CFO and the I/Q mismatch, so that turning OFF I/Q mismatch may reduce as well the impact of phase noise on the demodulator performance. The conclusions of 


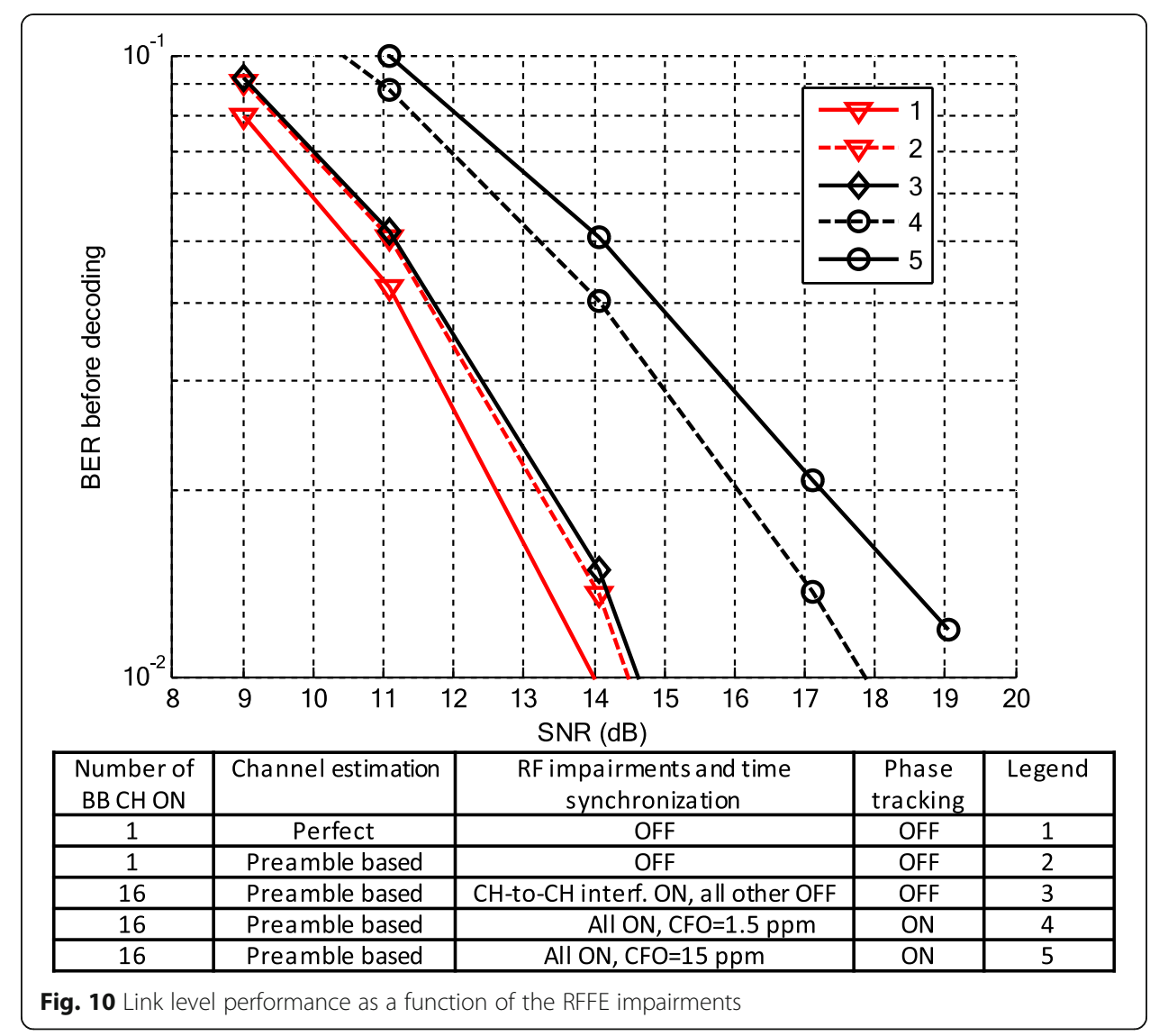

this analyst nevertheless give some clues about the dominant impairments from a qualitative point of view, but no quantitative conclusion can be definitively extracted from them. The results shown in Table 2 indicate that non-linearities on the Tx RF PAs and phase noise of the LO signals have a dominant contribution to the implementation loss, whereas other impairments such as LO spurs and channel-to-channel interferences at IF play a relatively minor role on the transceiver performance degradation.

\subsection{Validation using a real D band link and separate Tx and Rx LO generators}

Additionally, a proof of concept hardware platform shown in Fig. 11 has been set up for validating the signal processing and RF compensation algorithms on a real D band

Table 2 Implementation losses for PER $=10^{-5}$ when turning off individual impairments

\begin{tabular}{ll}
\hline Impairment & Implementation loss \\
\hline ALL ON & $6.0 \mathrm{~dB}$ \\
PAs non-linearities OFF & $4.25 \mathrm{~dB}$ \\
Phase noise OFF & $4.5 \mathrm{~dB}$ \\
LNAs and Mixers non-linearities OFF & $5.0 \mathrm{~dB}$ \\
CFO and I/Q mismatch OFF & $5.25 \mathrm{~dB}$ \\
LO spurs and channel-to-channel coupling OFF & $5.5 \mathrm{~dB}$ \\
Time synchronization OFF & $5.5 \mathrm{~dB}$ \\
ALL OFF & $0 \mathrm{~dB}$ \\
\hline
\end{tabular}


Table 3 Summary of transceiver and impairments parameter values

\begin{tabular}{|c|c|c|}
\hline \multicolumn{3}{|l|}{ Transceiver parameters } \\
\hline Number of BB channels & \multicolumn{2}{|l|}{16} \\
\hline Sampling frequency & \multicolumn{2}{|l|}{$2.64 \mathrm{GHz}$} \\
\hline Frame duration & \multicolumn{2}{|l|}{$30.3 \mu \mathrm{s}$} \\
\hline Signal bandwidth & \multicolumn{2}{|c|}{ 1.6 GHz per BB channel, $32 \mathrm{GHz}$ in total } \\
\hline Modulation & \multicolumn{2}{|l|}{16 QAM } \\
\hline Channel estimation & \multicolumn{2}{|c|}{ Preamble based } \\
\hline Time scattered pilots & \multicolumn{2}{|c|}{1 pilot every 16 samples } \\
\hline Cyclic prefix & \multicolumn{2}{|c|}{32 samples } \\
\hline Shaping filter & \multicolumn{2}{|c|}{ RRC, roll-off 0.17 order 30} \\
\hline Channel & \multicolumn{2}{|c|}{ Derived from [24] } \\
\hline Impairments parameters & ALL OFF & ALL ON \\
\hline Time synchronization & Perfect & Preamble based \\
\hline Tx power amplifiers & Linear & $\begin{array}{l}\text { Gain } 25 \mathrm{~dB}, \mathrm{P} 1 \mathrm{~dB} 12 \mathrm{dBm} \\
\text { Sat. power } 15 \mathrm{dBm}\end{array}$ \\
\hline Tx power amplifiers IBO & $\mathrm{N} / \mathrm{A}$ & $5 \mathrm{~dB}$ \\
\hline Rx LNA RF & Linear & Gain: 12 dB, IIP3: -25 dBm \\
\hline Rx RF mixers & Linear & Gain: 2 dB, IIP3: -28.6 dBm \\
\hline Rx IF LNAs & Linear & Gain: $16 \mathrm{~dB}$, IIP3: $-25 \mathrm{dBm}$ \\
\hline Rx IF LNBs & Linear & Gain: 14 dB, IIP3: -15 dBm \\
\hline Rx IF mixer & Linear & Gain: $6 \mathrm{~dB}$, IIP3: $-4.5 \mathrm{dBm}$ \\
\hline Total Rx NF & N/A & $10 \mathrm{~dB}$ \\
\hline LO spurious & N/A & $a_{1}=-20 \mathrm{~dB}, a_{2}=-20 \mathrm{~dB}$ \\
\hline Ch. to Ch. coupling at IF & N/A & $\beta_{1}=-20 \mathrm{~dB}, \beta_{2}=-23 \mathrm{~dB}, \beta_{3}=-26 \mathrm{~dB}$ \\
\hline Phase noise model & N/A & $\begin{array}{l}\text { Input ref }(2 \mathrm{GHz}): 1 / \mathrm{f}^{2} \mathrm{PN} @ 1 \mathrm{MHz} \text { offset }=-130 \mathrm{dBc} / \mathrm{Hz} \\
\text { Phase noise floors: } \\
\text { Input ref }=-150 \mathrm{dBc} / \mathrm{Hz} \\
\text { IF LOs }=-120 \mathrm{dBc} / \mathrm{Hz} \\
\text { RF LOs }=-120 \mathrm{dBc} / \mathrm{Hz}\end{array}$ \\
\hline
\end{tabular}

*See section 3.1

** See section 3.2

transmission and with hardware imperfections. The above-described signal processing DBB algorithms, shown in Fig. 9 for the Rx part, are simulated by a software (run with Matlab) and run on a PC that interfaces the instruments. The algorithms in the DBB Rx compensate some of the RF imperfections of the setup such as slow varying phase noise of the $\mathrm{LO}$ generators and CFO between the Tx and Rx LO generators, as well as channel equalization due to non-uniform gain across the signal bandwidth of the up and down converter heads and other $\mathrm{RF}$ elements (cables, attenuator between $\mathrm{Tx}$ and $\mathrm{Rx}$, etc.).

The DBB Tx generates the waveform that is sent to an arbitrary waveform generator (AWG), and frequency up-converted to D band by a sub-harmonic mixer VDI WR6.5, fed by a dedicated signal generator around $20 \mathrm{GHz}$ (6th order harmonic mixing). The RF signal is sent through an attenuator connecting the Tx and Rx (as shown in Fig. 11) or over the air using $10 \mathrm{dBi}$ horn antennas. The received signal is down converted by another sub-harmonic mixer to IF frequency using a separated LO generator. The signal is then sampled at IF by the oscilloscope and sent back to the DBB Rx code that carries out off-line processing on the received digitized signals. 


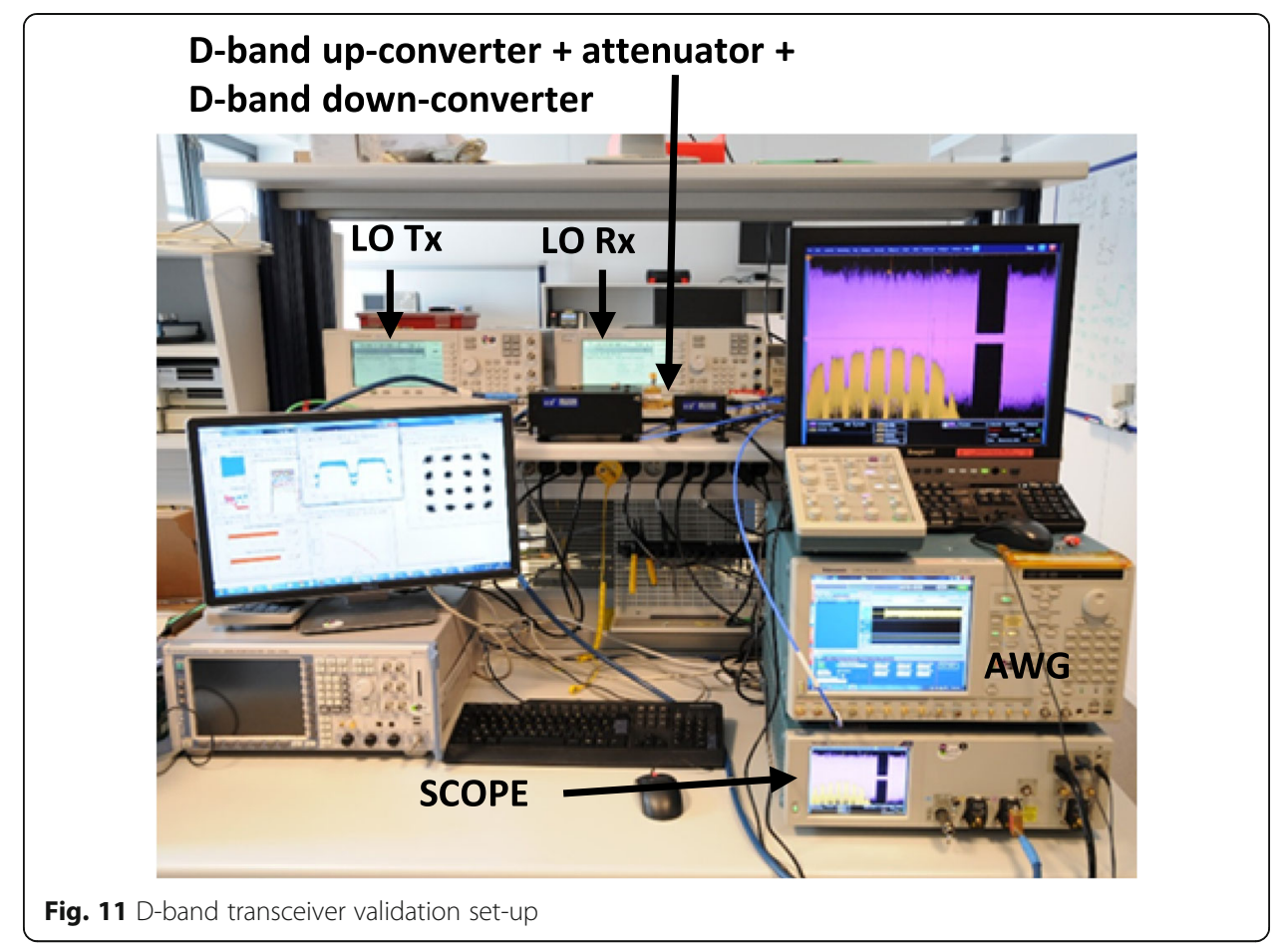

The digital to analog converters (DAC) in the AWG behave as a sinc filter, which limits the generated signal bandwidth. In the used AWG Tektronix 70001 offering 50 Gsamples/s, the first Nyquist zone occurs at $20 \mathrm{GHz}$, which allows to generate 8 channels of $2 \mathrm{GHz}$. The baseband signal computed in the simulation chain is first upsampled at the AWG sampling rate and digitally up converted at IF to become a real waveform centered into the AWG band. The data format is then converted to binary and exported to the AWG. The mean amplitude signal is set so that it optimizes a trade-off between the clipping and quantization noise of the AWG DACs on 10 bits. At the receiver side, the oscilloscope Tektronix DPO7000SX triggers on the first incoming frame, and acquires the signal at high sampling frequency (up to 200 Gsamples/s). The signal dynamic is optimized to fit the analog to digital converter (ADC) scale on 8 bits. The sampled signal is recorded and sent back to the software simulation chain for processing. The data is first digitally down converted from the receiver IF to baseband and down sampled to the simulation chain rate. The simulation chain provides the received signal constellation after synchronization, RF impairment compensations and channel equalization for every channel. The constellations shown in Fig. 12 are given as

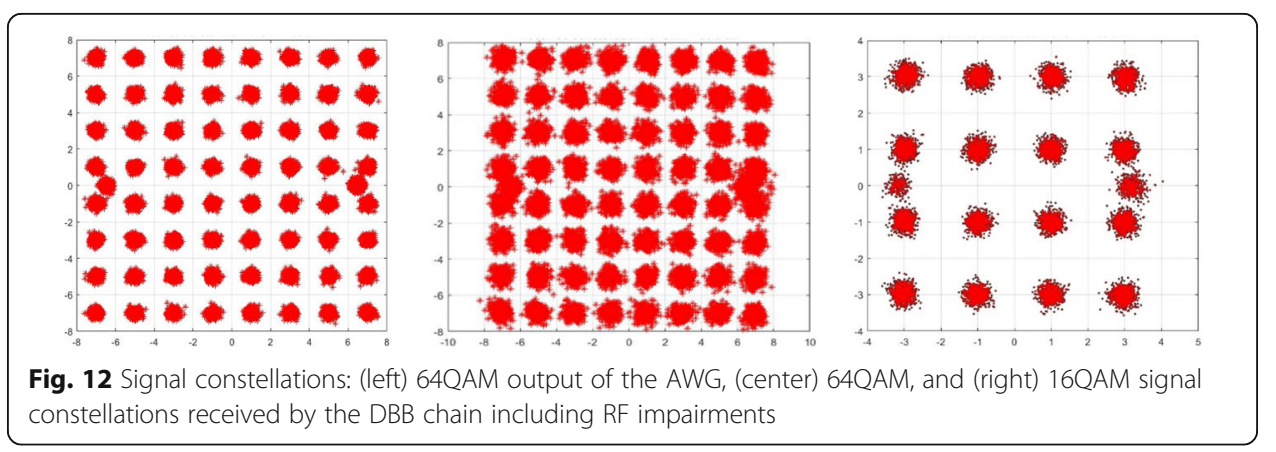


example for 64QAM, which is a worst case compared to the baseline, and 16QAM modulations. The received 64QAM constellation is compared with the ideal constellation from the simulation chain at the Tx side (generated by the AWG).

The received constellations exhibit a small rotation due to residual CFO and slowvarying phase noise. They have extra noise compared to the Tx modulation due to clipping, quantization, and channel overlapping. In all the figures, the BPSK modulated pilots are also visible on the constellation diagrams. For the 16QAM constellation, the impairments of the hardware platform degrade the SNR by $1.5 \mathrm{~dB}$ at BER before decoding of $10^{-3}$, compared to the ideal simulation chain without any impairment as shown in Fig. 13. No noise floor is observed at BER lower than $10^{-5}$.

The function of autocorrelation used for time synchronization is shown in Fig. 14 for various SNR values from 7 to $30 \mathrm{~dB}$. The algorithm achieves good time synchronization and coarse frequency estimation throughout all this range of SNR.

The estimation and compensation of the CFO is tested by changing the subharmonic up mixer LO frequency with respect to the down-converter mixer LO frequency. The algorithm achieves good estimation up to $20 \mathrm{ppm}$ CFO (which is the maximum CFO that can be corrected), with better than $0.5 \mathrm{ppm}$ precision (for SNR better than $7 \mathrm{~dB}$ ) and achieves perfect de-rotation and phase tracking as illustrated in Fig. 15.

\section{Conclusions}

This paper presents a $16 \mathrm{RF}$ channel-bonding transceiver architecture operating in D band able to provide $100 \mathrm{Gbps}$ of raw data rate for $50 \mathrm{~m}$ range. 16QAM modulation and digital baseband processing including RFFE impairments compensation has been

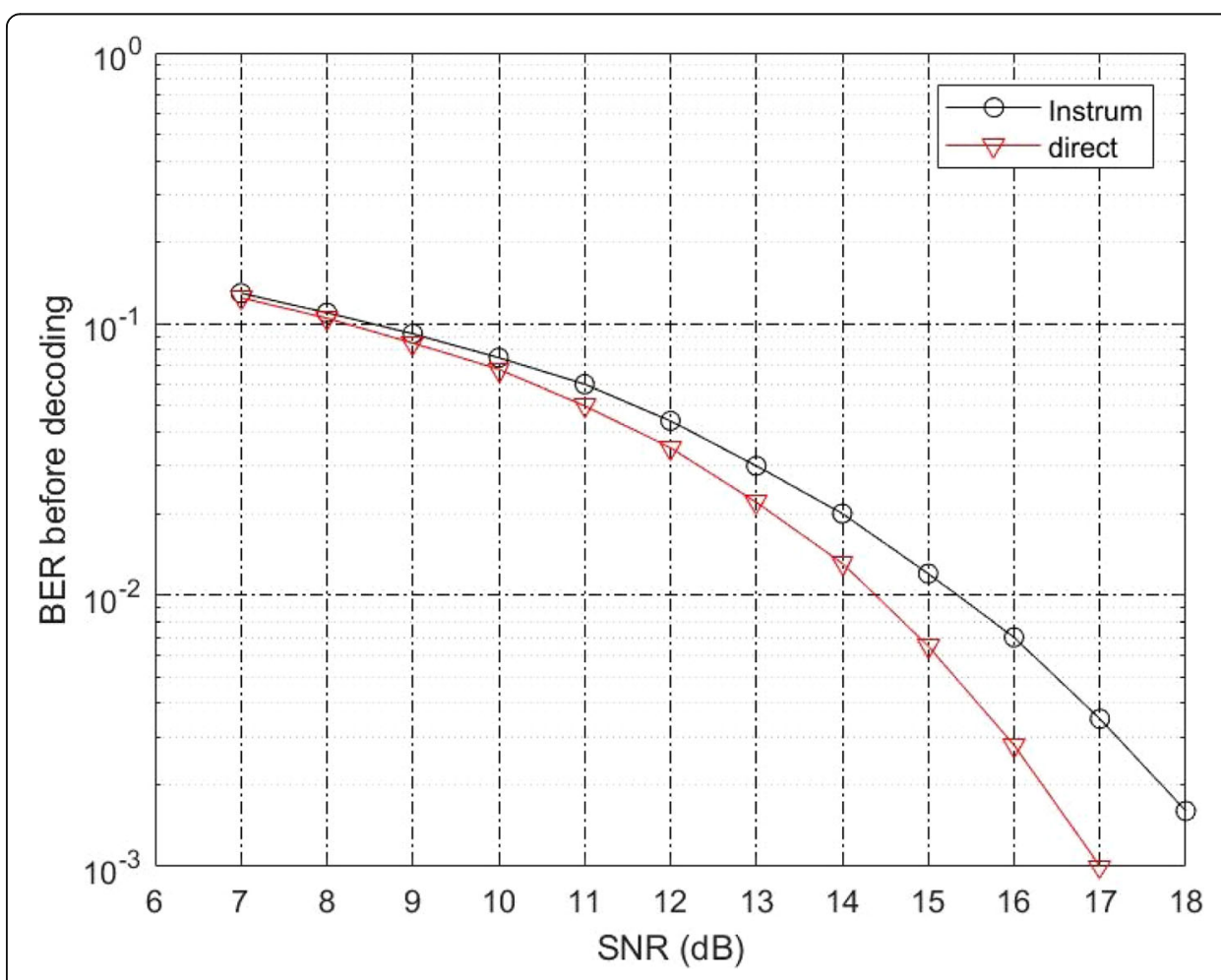

Fig. 13 Measured BER before decoding vs SNR for 16QAM 

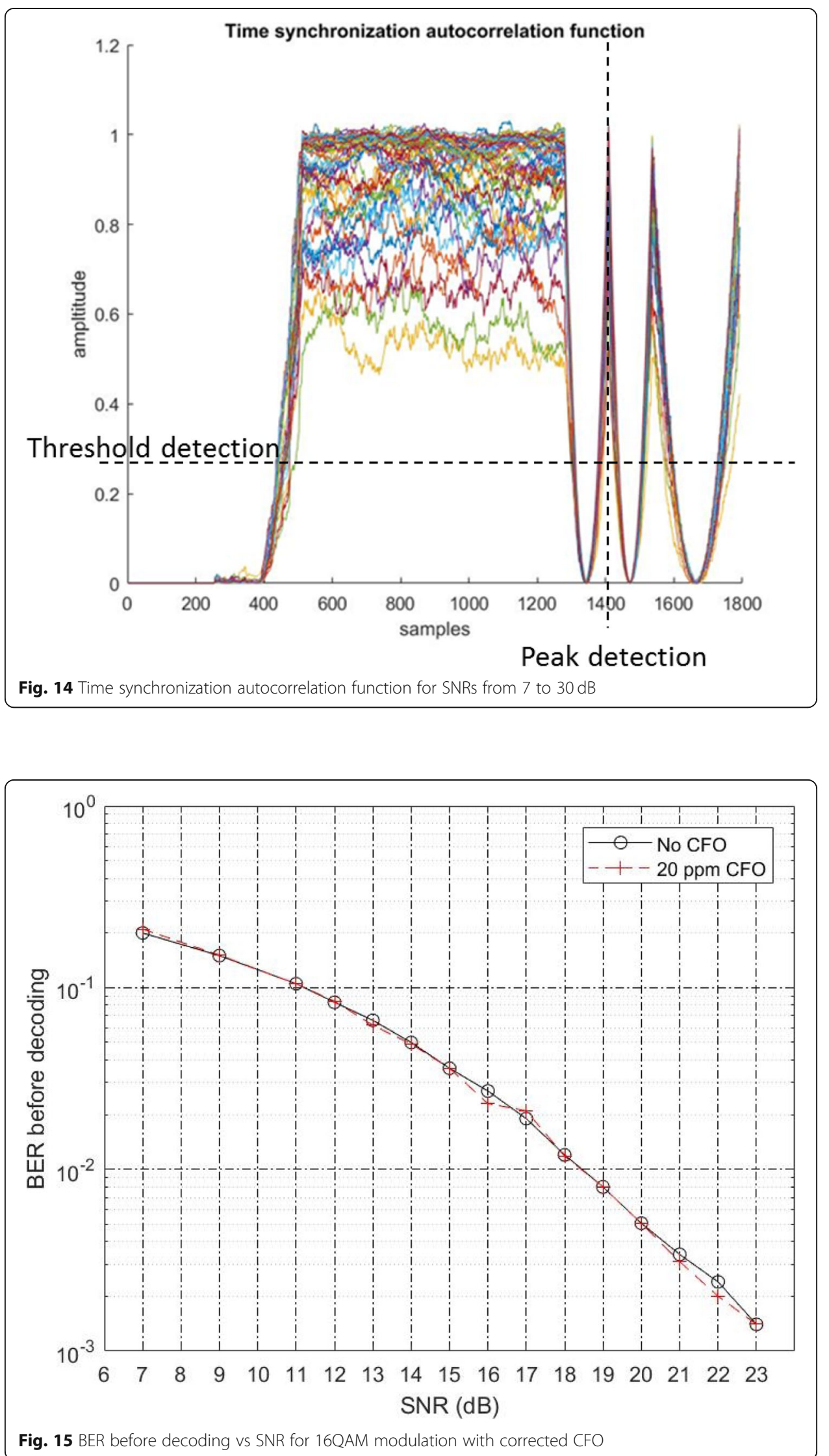

Fig. 15 BER before decoding vs SNR for 16QAM modulation with corrected CFO 
demonstrated. The RFFE is based on a double conversion architecture with multiple parallel lanes, and features a reduced number of LO frequencies. A full linklevel simulation platform including a realistic model of the channel has been developed and used to investigate the feasibility of the proposed architecture and to assess the performance of the DBB compensation algorithms. Phase noise and PA non-linearities have been found to be the most impacting non-idealities. Nevertheless, regarding phase noise, the proposed phase synchronization algorithm is able to cope with $\mathrm{LO}$ phase noise derived from an input signal at $2 \mathrm{GHz}$ with up to $-130 \mathrm{dBc} / \mathrm{Hz} @ 1 \mathrm{MHz}$ offset and a noise floor of $-154 \mathrm{dBc} / \mathrm{Hz}$ that translate to $-93 \mathrm{dBc} / \mathrm{Hz}$ and $-127 \mathrm{dBc} / \mathrm{Hz}$ at $140 \mathrm{GHz}$, respectively. Other performances such as $\mathrm{CFO}$ compensation and time synchronization have been also validated both using the link-level simulation platform with a realistic channel model and a hardware implementation, showing for example, that CFO between Tx and Rx of up to $20 \mathrm{ppm}$ can be tolerated without significant degradation. Both test beds (simulation and hardware) have been used to assess that channel-to-channel interferences and LO leakage at adjacent channels are not a limiting issue for this type of highly parallel RF architectures, paving the way to extremely large bandwidth mmW transceivers using relatively low base-band bandwidth.

\section{Abbreviations}

5G: 5th generation; ADC: Analog-to-digital converter; ASK: Amplitude shift keying; AWG: Arbitrary waveform generator; AWGN: Additive white Gaussian noise; BB: Base band; BER: Bit error rate; BiCMOS: Bipolar-complementary metal-oxidesemiconductor; BPSK: Binary phase shift keying; BW: Bandwidth; CFO: Carrier frequency offset; CMOS: Complementary metal-oxide-semiconductor; DBB: Digital base band; $\mathrm{dB}$ : Decibel; $\mathrm{dBc}$ : Power level in decibels relative to the carrier; $\mathrm{dBi}$ : Gain of antenna with respect to an isotropic source; dBm: Power units in decibel normalized to $1 \mathrm{~mW}$;

EIRP: Equivalent isotropic radiated power; eMBB: Enhanced mobile broadband; ETSI: European telecommunications standardization institute; FCC: Federal communications commission; FDE: Frequency domain equalization; Gbps: Gigabits per second; GHz: Gigahertz ( $10^{9}$ hertz); I/Q: In-phase and quadrature; IF: Intermediate frequency; IIP3: 3rd order input intercept point; ILO: Injection locked oscillator; kHz: Kilohertz (103 hertz); LNA: Low noise amplifier; LO: Local oscillator; MHz: Megahertz (10 hertz); MICl: Ministerio de Comercio e Industrias; mmW: Millimeter wave; NF: Noise figure; OCP1dB: $1 \mathrm{~dB}$ output compression point; OFDM: Orthogonal frequency division multiplexing; P1dB: $1 \mathrm{~dB}$ compression point; PA: Power amplifier; PAPR: Peak to average power ratio; PER: Packet error rate; PLL: Phased locked loop; PN: Phase noise; Pout: Output power; PSK: Phase shift keying; QAM: Quadrature amplitude modulation; QPSK: Quadrature phase shift keying; RF: Radio frequency; RFFE: Radio frequency front-end; RRC: Raised rooted cosine; Rx: Receiver; SC: Single carrier; SNR: Signal to noise ratio; Tx: Transmitter; WiGig: Name of a $60 \mathrm{GHz}$ standard for wireless gigabit; WPN: White phase noise

\section{Acknowledgements}

Not applicable

\section{Authors' contributions}

JL.G-J and DN have coordinated the work, JL.G-J, C.D., and A.S. have contributed with the modeling of the radio section and the experimental set-up, N.C., Y. D, A.dD., and V.S. have contributed with the design and implementation of the digital signal processing section of the transceiver, and A.C. and R.D'E have contributed with the air interface and channel modeling. All authors read and approved the final manuscript.

\section{Funding}

This work has been funded internally by CEA-Leti.

Availability of data and materials

Data sharing not applicable to this article as no datasets were generated or analyzed during the current study.

\section{Competing interests}

The authors declare that they have no competing interests.

Received: 29 July 2019 Accepted: 21 May 2020

Published online: 05 June 2020

\section{References}

1. Y. Xing, T. S. Rappaport, "Propagation measurement system and approach at $140 \mathrm{GHz}$-moving to $6 \mathrm{G}$ and above 100 GHz," in IEEE 2018 Global Communications Conference, Dec. 2018, pp.1-6. 
2. V. Dyadyuk et al., A multigigabit millimeter-wave communication system with improved spectral efficiency. IEEE Trans Microwave Theory Techniques 55(12), 2813-2821 (2007)

3. A. Hirata et al., 10-Gbit/s wireless link using InP HEMT MMICs for generating 120-GHz-band millimeter-wave signal. IEEE Trans Microwave Theory Techniques 57(5), 1102-1109 (2009)

4. C. Wang, C. Lin, Q. Chen, B. Lu, X. Deng, J. Zhang, A 10-Gbit/s wireless communication link using 16-QAM modulation in 140-GHz band. IEEE Trans Microwave Theory Techniques 61(7), 2737-2746 (2013)

5. I. Kallfass et al., All active MMIC-based wireless communication at $220 \mathrm{GHz}$. IEEE Trans Terahertz Sci Techn 1(2), 477-487 (2011)

6. J. Antes et al., $220 \mathrm{GHz}$ Wireless Data Transmission Experiments up to $30 \mathrm{Gbit} / \mathrm{S}, 2012$ IEEE/MTT-S International Microwave Symposium Digest (Montreal, QC, 2012), pp. 1-3

7. I. Kallfass et al., "MMIC chipset for $300 \mathrm{GHz}$ indoor wireless communication," 2015 IEEE International Conference on Microwaves, Communications, Antennas and Electronic Systems (COMCAS), Tel Aviv, 2015, pp. 1-4.

8. K.S. Kim, B. Kim, M. Kang, W. Byun, H.C. Park, 16-QAM OFDM-based W-band polarization-division duplex communication system with multi-gigabit performance. ETRI J. 36, 206-213 (2014)

9. L. Ohlsson and L. Wernersson, "A 15-Gb/s wireless ON-OFF keying link," in IEEE Access, vol. 2, pp. 1307-1313, 2014

10. V. Vassilev et al., "Spectrum efficient D-band communication link for real-time multi-gigabit wireless transmission," 2018 IEEE/MTT-S International Microwave Symposium - IMS, Philadelphia, PA, 2018, pp. 1523-1526.

11. E. Laskin, P. Chevalier, B. Sautreuil and S. P. Voinigescu, "A 140-GHz double-sideband transceiver with amplitude and frequency modulation operating over a few meters," 2009 IEEE Bipolar/BiCMOS Circuits and Technology Meeting, Capri, 2009, pp. 178-181.

12. S. V. Thyagarajan, S. Kang and A. M. Niknejad, "A 240GHz wideband QPSK receiver in $65 \mathrm{~nm}$ CMOS," 2014 IEEE Radio Frequency Integrated Circuits Symposium, Tampa, FL, 2014, pp. 357-360.

13. Y. Yang, S. Zihir, H. Lin, O. Inac, W. Shin and G. M. Rebeiz, "A 155 GHz 20 Gbit/s QPSK transceiver in 45 nm CMOS," 2014 IEEE Radio Frequency Integrated Circuits Symposium, Tampa, FL, 2014, pp. 365-368.

14. A. Balteanu, S. Shopov and S. P. Voinigescu, "A $2 \times 44 \mathrm{~Gb} / \mathrm{s} 110-\mathrm{GHz}$ wireless transmitter with direct amplitude and phase modulation in 45-nm SOI CMOS," 2013 IEEE Compound Semiconductor Integrated Circuit Symposium (CSICS), Monterey, CA, 2013, pp. 1-4.

15. K. Okada et al., "20.3 A 64-QAM 60GHz CMOS transceiver with 4-channel bonding," 2014 IEEE International Solid-State Circuits Conference Digest of Technical Papers (ISSCC), San Francisco, CA, 2014, pp. 346-347.

16. R. Wu et al., "13.6 A 42Gb/s 60GHz CMOS transceiver for IEEE 802.11 ay," 2016 IEEE International Solid-State Circuits Conference (ISSCC), San Francisco, CA, 2016, pp. 248-249.

17. K. K. Tokgoz et al., "13.3 A 56Gb/s W-band CMOS wireless transceiver," 2016 IEEE International Solid-State Circuits Conference (ISSCC), San Francisco, CA, 2016, pp. 242-243.

18. S. Shopov, O. D. Gurbuz, G. M. Rebeiz and S. P. Voinigescu, "A 13.2-dBm, 138-GHz I/Q RF-DAC with 64-QAM and OFDM free-space constellation formation," ESSCIRC 2017 - 43rd IEEE European Solid State Circuits Conference, Leuven, 2017, pp. 191-194.

19. S. Jia et al., "120 Gb/s multi-channel THz wireless transmission and THz receiver performance analysis," in IEEE Photonics Technology Letters, vol. 29, no. 3, pp. 310-313, 1 Feb.1, 2017.

20. K. Okada et al., Millimeter-Wave CMOS Transceiver toward 1Tbps Wireless Communication, Asia-Pacific Microwave Conference (2018)

21. P. Rodríguez-Vázquez, J. Grzyb, B. Heinemann, U.R. Pfeiffer, A 16-QAM 100-Gb/s 1-M wireless link with an EVM of 17\% at $230 \mathrm{GHz}$ in an SiGe technology. IEEE Microwave and Wireless Components Letters (2019)

22. K. Liu et al., "100 Gbit/s THz photonic wireless transmission in the $350-\mathrm{GHz}$ band with extended reach," in IEEE Photonics Technology Letters, vol. 30, no. 11, pp. 1064-1067, 1 June1, 2018.

23. S. Jia et al., $120 \mathrm{~Gb} / \mathrm{S}$ Multi-Channel THz Wireless Transmission and THz Receiver Performance Analysis, IEEE Photonics Technology Letters (2017)

24. L. Pometcu, R. D'Errico, Characterization of Sub-THz and Mmwave Propagation Channel for Indoor Scenarios, 12th European Conference on Antennas and Propagation (EuCAP 2018) (2018)

25. B. Murmann, "ADC performance survey 1997-2020," [Online]. Available: http://web.stanford.edu/ murmann/adcsurvey.html.

26. C. Jany, A. Siligaris, J. L. Gonzalez-Jimenez, P. Vincent and P. Ferrari, "A programmable frequency multiplier-by-29 architecture for millimeter wave applications," in IEEE Journal of Solid-State Circuits, vol. 50, no. 7, pp. 1669-1679, July 2015.

27. D. Simic and P. Reynaert, "A $14.8 \mathrm{dBm} 20.3 \mathrm{~dB}$ Power amplifier for D-band applications in $40 \mathrm{~nm}$ CMOS," 2018 IEEE Radio Frequency Integrated Circuits Symposium (RFIC), Philadelphia, 2018, pp. 232-235.

28. F. F. Manzillo, A. Clemente, B. Blampey, G. Pares, A. Siligaris and J. Luis González-Jiménez "Transmitarray Antenna with Integrated Frequency Multiplier for High-speed D-band Communications in Low-cost PCB Technology," 2019 13th European Conference on Antennas and Propagation (EuCAP), Krakow, 2019, pp. 1-4.

29. Abdelaziz Hamani, Alexandre Siligaris, Benjamin Blampey, Cedric Dehos, Jose Luis Gonzalez Jimenez, "A 125.5-157 GHz 8 dB NF and $16 \mathrm{~dB}$ of gain D-band low noise amplifier in CMOS SOI 45 nm," 2020 IEEE International Microwave Symposium (IMS), pp. 1-3, 2020.

30. A. Siligaris et al., "A $65-\mathrm{nm}$ CMOS fully integrated transceiver module for $60-\mathrm{GHz}$ wireless HD applications," in IEEE Journal of Solid-State Circuits, vol. 46, no. 12, pp. 3005-3017, 2011.

31. F. Barrera, A. Siligaris, B. Blampey and J. L. Gonzalez-Jimenez, "A D-band 4-ways power splitter/combiner implemented on a $28 \mathrm{~nm}$ bulk CMOS process," 2019 49th European Microwave Conference (EuMC), Paris, France, 2019, pp. 868-871.

32. L. Pometcu, R. D'Errico, "Characterization of sub-THz and mmwave propagation channels for indoor scenarios," in 12th Eurepean Conference on Antennas and Propagation, 2018.

33. C. Dehos, Des Noes M and D. Morche, "Sensitivity of MC-CDMA systems to carrier phase noise : a large system analysis," 2005 IEEE 16th International Symposium on Personal, Indoor and Mobile Radio Communications, Berlin, 2005, pp. 407-411. 
34. Tubbax, J., Fort, A., Van der Perre, L., Donnay, S., Engels, M., Moonen, M., \& De Man, H., "Joint compensation of IQ imbalance and frequency offset in OFDM systems, " In Global Telecommunications Conference, Vol. 4, pp. 2365-2369, 2003.

35. Martineau, B.; Knopik, V.; Siligaris, A.; Gianesello, F.; Belot, D.; , "A 53-to-68GHz 18dBm power amplifier with an 8-way combiner in standard $65 \mathrm{~nm}$ CMOS," 2010 IEEE international solid-state circuits conference digest of technical papers (ISSCC), pp. 428-429, 2010

36. Fabrizio Pancaldi, Giorgio M. Vitetta, Reza Kalbasi, Naofal Al-Dhahir, Murat Uysal, and Hakam Mheidat, "Single-carrier frequency domain equalization," IEEE Signal Processing Magazine, Vol. 37, 2008.

\section{Publisher's Note}

Springer Nature remains neutral with regard to jurisdictional claims in published maps and institutional affiliations.

Submit your manuscript to a SpringerOpen ${ }^{\odot}$ journal and benefit from:

- Convenient online submission

- Rigorous peer review

- Open access: articles freely available online

- High visibility within the field

- Retaining the copyright to your article

Submit your next manuscript at $\boldsymbol{\nabla}$ springeropen.com 